Check for updates

Cite this: RSC Adv., 2017, 7, 19250

\title{
Colorimetric detection of cholic acid based on an aptamer adsorbed gold nanoprobe $\uparrow$
}

\author{
Qiuyun Zhu, Tingting Li, \$\$ Yi Ma, \$ Zhaohui Wang, Jinxin Huang, Ruonan Liu \\ and Yueqing $\mathrm{Gu}^{*}$
}

Cholic acid (CA) has been proved as an effective clinical biomarker for disease diagnosis and treatment monitoring. However, traditional CA detection methods are tedious, time-consuming and expensive, and the results cannot be visualized. Herein, we present a facile, low-cost, fast and label-free biosensor platform based on a CA aptamer (48 nt) modified gold nanoprobe for CA detection. CA can bind with the surface-adsorbed CA aptamers competitively and detach the aptamer from the gold nanoparticles (Au NPs). Au NPs modified with aptamers of different densities will grow into morphologically varied nanostructures. The result can be observed visibly and quantified easily with the localized surface plasmon resonance (LSPR) spectra. The detection limit (LOD) was $1 \mu \mathrm{M}$, which was lower than the existing colorimetric detection methods based on Au NPs. Furthermore, the affinity between CA and 48 nt aptamer was verified for the first time by MicroScale Thermophoresis (MST) technology.

Received 7th January 2017

Accepted 12th March 2017

DOI: $10.1039 / \mathrm{c} 7 \mathrm{ra00255f}$

rsc.li/rsc-advances

Localized surface plasmon resonance (LSPR) based biosen-

\section{Introduction}

Bile acid, the major metabolite of cholesterol, is a mixture of steroids, and plays an important physiological role in the elimination of cholesterol from the body and in facilitating the absorption of dietary lipids and fat-soluble vitamins by formation of micelles. ${ }^{\mathbf{1} 2}$ The concentration of bile acids in the body is related to hepatitis, gallstones and other diseases. It has been shown that the concentration of bile acids in individuals with liver and intestinal diseases varies from 67 to $376 \mu \mathrm{mol} \mathrm{L}^{-1}$, while in healthy individuals the range is from 4 to $10 \mu \mathrm{mol}$ $\mathrm{L}^{-1.3,4}$ Additionally, bile acids also have pharmacological activity, and some components of bile acids have important therapeutic applications in treating some diseases, such as primary biliary cirrhosis and cholesterol gallstones., ${ }^{5,6}$ Cholic acid (CA) comprises $31 \%$ of the total bile acids (TBA) produced in the liver. The concentration of cholic acid has been proved as an effective indicator in the diagnosis of liver and intestinal diseases. $^{7}$ Hence, the quantification of bile acids, especially cholic acid is vital for diseases diagnosis and treatment monitoring. Traditional cholic acid detection involves spectroscopic and chromatographic methods such as HPLC and LC-MS, which are tedious, time-consuming, expensive and the results cannot be visualized.

Department of Biomedical Engineering, School of Engineering, China Pharmaceutical University, Nanjing 210009, China.E-mail: guengineering@cpu.edu.cn

$\dagger$ Electronic supplementary information (ESI) available: Synthesis, photo physical, and experiments additional figures and images (Fig. S1-S11). See DOI: 10.1039/c7ra00255f

\$ These authors contributed equally. sors are superior for in vitro detections (IVDs) because these methods are cost-effective, fast, simple and highly sensitive. ${ }^{\mathbf{8 9}}$ Gold nanoparticles (Au NPs) have been recognized as a promising candidate for LSPR-based IVDs due to ultrahigh extinction coefficients, easy synthesis, tunable surface modification, favorable stability and biosafety. ${ }^{\mathbf{1 0 , 1 1}}$ Through the precise control of the aggregation, shape and size, the LSPR absorption of Au NPs can be tuned, which is usually accompanied by color changes from red to purple or blue. ${ }^{12-15}$ Nucleic acid aptamers have attracted much attention over the past decades as a promising alternative to antibodies for IVD sensors. They are thermostable, fold reversible, inexpensive and can be readily synthesized via standard laboratory techniques such as PCR. ${ }^{16-18}$ Currently, novel LSPR biosensors based on aptamerAu NPs probes have been extensively investigated. ${ }^{19-21}$ However, such sensing platforms are mostly based on Au NPs aggregation and can be easily affected by biological environment. Recently, $\mathrm{Lu}$ et al. demonstrated for the first time that single-stranded DNA (ssDNA) could be used to tune Au NPs morphology during crystal growth. ${ }^{22}$ Based on this principle, Stevens et al. first reported a novel colorimetric assay that couples aptamertarget recognition and aptamer-mediated control of Au NPs morphology for the sensitive and naked-eye detection of ochratoxin A, cocaine and $17 \beta$-estradiol. ${ }^{23}$

In this study, we designed a simple and fast aptamer-Au NPs biosensor for the in vitro naked eye detection of cholic acid. Cholic acid aptamers (48 nt) were adsorbed on Au NPs surface via $\mathrm{Au}$-nucleoside affinity and charge-shielding effect of $\mathrm{MgCl}_{2}$. Cholic acid can regulate amounts of ssDNA strands from the Au NPs surface by aptamer-target interaction, which could affect 
the morphology of grown Au NPs, consequently producing solutions of different colors. Furthermore, we confirm the binding affinity of CA and the aptamer by MicroScale Thermophoresis (MST) for the first time and investigated the desorption process using FAM-labeled aptamer by fluorescence resonance energy transfer (FRET).

\section{Experimental section}

\section{Materials and reagents}

Chloroauric acid $\left(\mathrm{HAuCl}_{4}\right)$ was purchased from Guoyao Reagent Corporation (Shanghai, China). Sodium citrate $\left(\mathrm{Na}_{3} \mathrm{C}_{6} \mathrm{H}_{5} \mathrm{O}_{7}\right)$, magnesium chloride $\left(\mathrm{MgCl}_{2}\right)$ and 5(6)-carboxyfluorescein (FAM) were purchased from Sinopharm Chemical Reagent Co., Ltd. (China). Hydroxylammonium chloride $\left(\mathrm{NH}_{2} \mathrm{OH} \cdot \mathrm{HCl}\right)$ and dimethylsulfoxide (DMSO) were obtained from Nanjing Chemical Reagent Co., Ltd. (China). Cholic acid (CA), deoxycholic acid (DCA), chenodeoxycholic acid (CDCA), glycocholic acid (GCA) and lithocholic acid (LCA) were purchased from Sigma-Aldrich Inc. (USA). All chemical reagents were analytical grade and were used without further purification. All solutions were prepared using deionized water (Milli-Q grade, Millipore) with a resistivity of $18.2 \mathrm{M} \Omega \mathrm{cm}$.

The following sequences were synthesized by Sangon Biological Engineering Technology \& Co. Ltd (Shanghai, China):

CA aptamer: 5'-GCA GGG TCA ATG GAA TTA ATG ATC AAT TGA CAG ACG CAA GTC TCC TGC-3'; CA aptamer complementary sequence: $5^{\prime}$-GCA GGA GAC TTG CGT CTG TCA ATT GAT CAT TAA TTC CAT TGA CCC TGC-3'; FAM-labeled CA aptamer: 5'-FAM-GCA GGG TCA ATG GAA TTA ATG ATC AAT TGA CAG ACG CAA GTC TCC TGC-3'.

\section{Preparation of Au NPs}

Au NPs ( $\sim 15 \mathrm{~nm}$ in diameter) were prepared by citrate reduction method. By quickly adding $10 \mathrm{~mL}$ trisodium citrate $(38.8 \mathrm{mM})$ to a boiling $100 \mathrm{~mL} \mathrm{HAuCl}_{4}$ solution $(1 \mathrm{mM})$ with vigorous stirring, and the reaction last $30 \mathrm{~min}$. Until the color turned to deep red, the solution was centrifuged at $5000 \mathrm{rpm}$ for $5 \mathrm{~min}$, and the supernatant was stored at $4{ }^{\circ} \mathrm{C}$ until use.

\section{Preparation of Apt-Au NPs and growth analysis}

Amounts stated here refer to the preparation of Apt-Au NP from a single well (volume of $180 \mu \mathrm{L}$ ) in the 96-well plate (Corning, USA) and can be scaled up depending on the number of wells required. The facile synthesis of the Apt-Au NP probe was as follows, different volumes $(0,2.5,5,7.5,10,12.5$ and $15 \mu \mathrm{L})$ of CA aptamer $(2 \mu \mathrm{M})$ were added to given amounts $(8,16$ and 24 $\mu \mathrm{L})$ of $\sim 15 \mathrm{~nm} \mathrm{Au} \mathrm{NPs}(5 \mathrm{nM})$, with $20 \mu \mathrm{L}$ of $\mathrm{MgCl}_{2}(1 \mathrm{mM})$, then supplementary water was added to $180 \mu \mathrm{L}$. Adsorption reactions were conducted overnight with gently shaking at room temperature. Finally, the Apt-Au NP probe was acquired.

For the growth of Apt-Au NPs, $\mathrm{NH}_{2} \mathrm{OH} \cdot \mathrm{HCl}(5 \mu \mathrm{L}, 167 \mathrm{mM})$ and $\mathrm{HAuCl}_{4}(10 \mu \mathrm{L}, 2 \mathrm{mM})$ were sequentially added to the prepared probe solution. Another two aliquots of $\mathrm{HAuCl}_{4}$ were then added with about 5 min incubation between each addition. The solutions were mixed thoroughly using the pipet while adding to each well. The color change and UV-vis spectra of solutions were recorded with a camera and a POLARstar Omega multi-mode microplate reader (BMG LABTECH, Germany).

\section{Characterizations}

The morphology of the prepared Au NPs and Apt-Au NP were characterized by JEOL JEM-2100 transmission electron microscopy (TEM). The nanostructures with same concentrations of Au NPs were subjected to dynamic light scattering (Zetaplus nanoparticle size analyzer, Brookhaven) to measure the hydrodynamic diameter and zeta potential. An OD-1000+ UV-Vis spectrophotometer (One Drop ${ }^{\circledR}$ Technologies, China) was used for the detection of the absorption spectra of the aptamer, $\mathrm{Au}$ NPs and the Apt-Au NP probe.

\section{Interaction analysis of CA and CA aptamer}

The concentrations of FAM labeled aptamer and FAM solution (negative control) were both kept constant at a concentration of $0.1 \mu \mathrm{M}$ in MST optimized buffer (50 mM Tris-HCl buffer, pH 7.6 containing $150 \mathrm{mM} \mathrm{NaCl}, 10 \mathrm{mM} \mathrm{MgCl}$ and 0.05\% Tween-20). The unlabeled binding partners (CA) were titrated in $1: 1$ dilutions beginning at a final concentration of $500 \mu \mathrm{M}$ with MST optimized buffer containing 4\% DMSO. After 1 : 1 mixing, the samples were incubated for $20 \mathrm{~min}$ and filed into standard treated capillaries (Cat\#K002, NanoTemper Technologies, Germany). Next, a primary capillary scan determined the exact position of the capillaries on the holder and gave information on fluorescence quenching as well as the solubility of the aptamer and CA-aptamer complex. The measurements were conducted on a NanoTemper Monolith NT.115 instrument. And the analysis was performed at 20\% "LED Power" and 40\% "MST Power", "Fluo. Before" time was $5 \mathrm{~s}$, "MST on" time was $30 \mathrm{~s}$ and "Fluo. After" time was $5 \mathrm{~s}$. Normalized fluorescence versus CA concentrations were analyzed using the NanoTemper analysis software (NTAnalysis_v1.5.41). $K_{\mathrm{d}}$ values were calculated according to the following equation (law of mass action): $f(c)=$ unbound $+($ bound - unbound $) / 2 \times\left(\right.$ FluoConc $+c+K_{\mathrm{d}}-$ $\operatorname{Sqrt}\left(\left(\text { FluoConc }+c+K_{\mathrm{d}}\right)^{2}-4 \times\right.$ FluoConc $\left.\left.\times c\right)\right)$ where $f(c)$ is the fluorescence intensity of the mixed solution of CA and aptamer; bound is the number of moles of the bounding of CA and aptamer; unbound is the number of moles of the unbounding of CA and aptamer; FluoConc is the concentration of FAM-apt; $c$ is the concentration of CA; $K_{\mathrm{d}}$ is the affinity constant of CA and aptamer.

\section{Targets detection in solution}

DCA, CDCA, GCA and LCA were prepared in DMSO with concentrations of $100 \mathrm{mM}$. Then the solutions were respectively diluted to the required concentration $(1,10,50,100,300 \mu \mathrm{M})$ with double distilled water as samples. Different concentrations of samples $(20 \mu \mathrm{L})$ were added to $180 \mu \mathrm{L}$ of Apt-Au NP probes in each well respectively followed by incubation for $30 \mathrm{~min}$. The $\mathrm{Au}$ NPs solution, containing $\mathrm{MgCl}_{2}(20 \mu \mathrm{L}, 1 \mathrm{mM})$, was served as the negative control group. Then $\mathrm{NH}_{2} \mathrm{OH} \cdot \mathrm{HCl}(5 \mu \mathrm{L}, 167 \mathrm{mM})$ and different aliquots of $\mathrm{HAuCl}_{4}(10 \mu \mathrm{L}, 2 \mathrm{mM})$ were sequentially added to each well in every 5 min with sufficient mixing. The 
color change and UV-vis spectra of solutions were recorded with a camera and a POLARstar Omega multi-mode microplate reader (BMG LABTECH, Germany).

\section{Aptamer desorption analysis}

$4 \mu \mathrm{L}$ of FAM-modified CA aptamer $(2 \mu \mathrm{M})$ was added to $16 \mu \mathrm{L}$ of $\mathrm{Au}$ NPs $(15 \mathrm{~nm}, 5 \mathrm{nM}), 20 \mu \mathrm{L}$ of $\mathrm{MgCl}_{2}(1 \mathrm{mM})$, and $140 \mu \mathrm{L}$ of water. Then the mixture was reacted overnight in dark. Then $20 \mu \mathrm{L}$ of CA solutions at various concentrations were added and incubated at room temperature for $30 \mathrm{~min}$. After centrifugation (16 $000 \mathrm{rpm}, 10$ $\mathrm{min})$, the fluorescence of the supernatant was measured $\left(\lambda_{\mathrm{ex}}=485\right.$ $\pm 12 \mathrm{~nm}, \lambda_{\mathrm{em}}=520 \pm 10 \mathrm{~nm}$ ) using a POLARstar Omega multimode microplate reader (BMG LABTECH, Germany).

\section{Statistical methods}

MST data were processed by NT Analysis 1.5.41 software. Descriptive statistical analyses were performed using Origin 8.0 for calculating the means and the standard error of the mean. Results were expressed as the mean \pm standard deviation (SD).

\section{Results and discussions}

\section{Fabrication and characterizations of Apt-Au NP}

The CA aptamers were synthesized by Sangon Biological Engineering Technology \& Co. Ltd (Shanghai, China). The $T_{\mathrm{m}}$ of the aptamer was $70.67^{\circ} \mathrm{C}$. The aggregate MW was detected by Thermo LCQ Deca XP and the result was shown in Fig. S1. $\dagger$ Au NPs were prepared by citrate reduction method at first. Then, CA aptamers and $\mathrm{Au}$ NPs were linked together via physical adsorption of $\mathrm{Au}-$ nucleoside affinity (Fig. 1a). ${ }^{24,25}$ During the synthetic procedures of the Apt-Au NP probe, $\mathrm{Mg}^{2+}$ played a vital role in the absorption of ssDNA to negatively charged Au NPs surfaces, as the grown solution of Apt-Au NPs turned purplish red with $\sim 10 \mathrm{~nm}$ LSPR shift in the absence of $\mathrm{Mg}^{2+}$, which was negligible in the group adding $\mathrm{Mg}^{2+}$ (Fig. S2 $\dagger$ ). Hence, we supposed that moderate $\mathrm{Mg}^{2+}$ could facilitate the adsorption process by charge-shielding effect. TEM
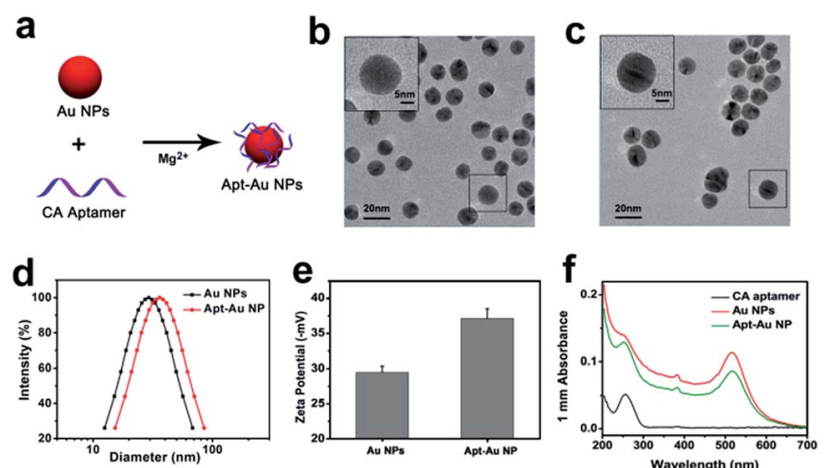

Fig. 1 Characterizations of the prepared Au NPs and the Apt-Au NP probe. (a) Schematic of adsorption process with $A u$ NPs and CA aptamer via Au-ssDNA affinity and charge-shielding effect mediated by $\mathrm{Mg}^{2+}$. (b) TEM images of Au NPs and (c) the Apt-Au NP probe, scale bars $=20 \mathrm{~nm}$ and the inner scale bars $=5 \mathrm{~nm}$. (d) Hydrodynamic diameters of Au NPs and Apt-Au NP. (e) Zeta potential of Au NPs and Apt-Au NP. (f) UV-vis spectra of CA aptamer, Au NPs and Apt-Au NP. images showed that both of Au NPs (Fig. 1b) and Apt-Au NP (Fig. 1c) were spherical and monodisperse with an average size of $\sim 15 \mathrm{~nm}$. Compared with Au NPs, a thin halo layer was observed around Apt-Au NP from the enlarged image, which was indicative of a successful aptamer adsorption. However, there was a slight particle aggregation in Fig. 1c, which was caused by extra addition of $\mathrm{Mg}^{2+}(0.25 \mathrm{mM})$. DLS data showed that the hydrodynamic diameters of Au NPs and Apt-Au NP were $29.2 \pm 0.2 \mathrm{~nm}$ and 36.1 $\pm 1.3 \mathrm{~nm}$ respectively (Fig. 1d), and the addition of ssDNA aptamers caused the zeta-potential of Au NPs (Fig. 1e) to change from $-29.5 \pm 0.91 \mathrm{mV}$ to $-37.2 \pm 1.4 \mathrm{mV}$ (without CA aptamer adsorption), indicating that the negatively charged nucleic acid has stranded onto the surface of Au NPs. Moreover, the absorption spectra showed that Apt-Au NP possessed the same absorption peak at $520 \mathrm{~nm}$ with the bare Au NPs and $260 \mathrm{~nm}$ with the ssDNA aptamers (Fig. 1f). The above results, therefore, indicated the successful preparation of CA aptamers modified Au NPs.

Adsorption of ssDNA on Au NPs is well known for enhancing the electrostatic repulsion between Au NPs and preventing them from salt-induced aggregation. ${ }^{26,27}$ Hence, we incubated CA aptamer sequences with Au NPs, and then challenged the Au NPs and Apt-Au NP solution with $\mathrm{NaCl}(25 \mathrm{mM}$ and $50 \mathrm{mM})$. As shown in Fig. S3, $\uparrow$ aggregation of bare Au NPs happened immediately with color change and there was a platykurtic UVvis absorption spectrum at $25 \mathrm{mM} \mathrm{NaCl}$ solution. The solution turned colorless with visible particulate suspended and the LSPR of Au NPs was faded as $\mathrm{NaCl}$ increased to $50 \mathrm{mM}$, while $\mathrm{Au}$ NPs incubated with CA aptamer sequences remained stable and maintained their native burgundy color. Since the stability of the Au NPs was enhanced at salt solution by adsorption of ssDNA on its surface.

\section{Binding of CA to CA aptamer}

In 2000, Kato ${ }^{28}$ had screened several DNA aptamers which bind to CA from a pool of approximately $9 \times 10^{14}$ DNA molecules by in vitro selection. And the affinity of CA with aptamers was analyzed by the equilibrium-filtration method. Results suggested that the ssDNA with 48 nucleotide length was a better alternative for binding to cholic acid immobilized selection column due to the highest affinity $\left(K_{\mathrm{d}}=5 \mu \mathrm{M}\right)$ and the relatively shorter nucleotide length. We used MST to further validate the binding affinity between CA and aptamer because of its robust and fast analysis. FAM modified CA aptamers were used at a constant concentration of $0.1 \mu \mathrm{M}$, and judging from 14 capillaries scan which was carried out before each MST measurement. Any aggregation or strong non-specific binding to the capillary could probably be excluded as it would have yielded a different peak shape (Fig. S4a†). Then we tested the interaction of aptamer with CA. The binding curve was shown in Fig. S4b $\dagger$ and the shape of the MST curves for the FAM labeled aptamer-CA interaction with a calculated $K_{\mathrm{d}}$ of $12.6 \pm 0.695 \mu \mathrm{M}$ (Fig. 2). However, to ensure that no FAM effects contributed to the binding processes, CA was further co-incubated with FAM to analyze the potential interaction, but no apparent binding was observed from the curve. Above all, the specific binding capacity of the CA molecule to the aptamer was fully vindicated, which 


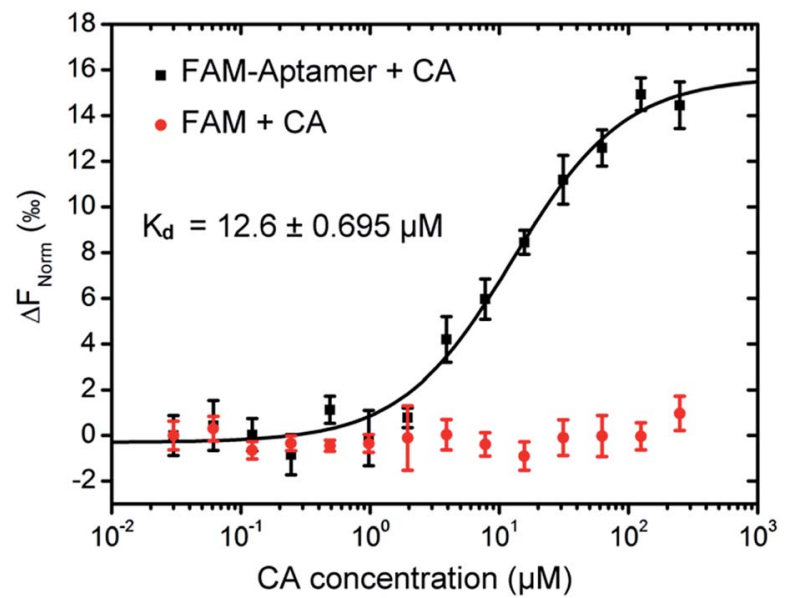

Fig. 2 Binding of CA to FAM labeled aptamers and FAM. The concentration of the FAM-Apt was kept constant at $0.05 \mu \mathrm{M}$, while the concentration of CA was varied from 0.03-250 $\mu \mathrm{M}$. Error bars indicate the SD of three independent experiments.

also implied that in the presence of CA, the aptamer-CA interaction could result in the desorption of aptamers from the surface of Au NPs.

\section{Preparation of Apt-Au NP probe}

The amount of adsorbed aptamers remaining on the Au NP surface was vital for the morphology change of grown Au NPs and was a highly sensitive barometer of the CA concentration. During the growth analysis of Apt-Au NPs, we found that twice addition of $\mathrm{HAuCl}_{4}$ could achieve better color differentiation with limited aptamers concentration (Fig. S5 $\dagger$ ). In addition, to obtain a largest LSPR shift of Apt-Au NP probes at the LOD, amounts of added Au NPs and ssDNA were further investigated. As evident in Fig. 3a, after treated with $\mathrm{NH}_{2} \mathrm{OH} \cdot \mathrm{HCl}$ and twice addition of $\mathrm{HAuCl}_{4}$ to proceed the growth reaction, increasing amounts of adsorbed aptamers gave rise to color change from red to blue, and a red shift in the UV-vis spectra of grown Au NPs was observed (Fig. 3b). Notably, a higher Au NPs concentration led to the requirement of more CA aptamer at the same LSPR shift of the grown Au NPs. As the aptamers less than $150 \mathrm{nM}$, an obvious color gradient was seen with addition of $0.4 \mathrm{nM}$ Au NPs compared with other concentrations, and corresponded to a peak wavelength $590 \mathrm{~nm}$, where the solution turned blue and remained constant. Therefore, we used $0.4 \mathrm{nM}$ Au NPs as a better parameter for fabrication of Apt-Au NPs to detect CA in vitro. Additionally, increasing amounts of adsorbed CA aptamer gave rise to a more irregular shape of grown Au NPs from TEM images (Fig. 3c). These results have shown that grown Apt-Au NP solutions were lyons blue or deep blue when ratios of Au NPs and aptamer were $1: 250,1: 312.5$ and $1: 375$. Therefore, we chose the case of $1: 250$ to prepare the probe, which can produce the largest LSPR shift at LOD.

\section{Detection of CA with Apt-Au NP probe}

Currently, there are few methods for the detection of bile acids, especially cholic acid. Based on the principle of gold
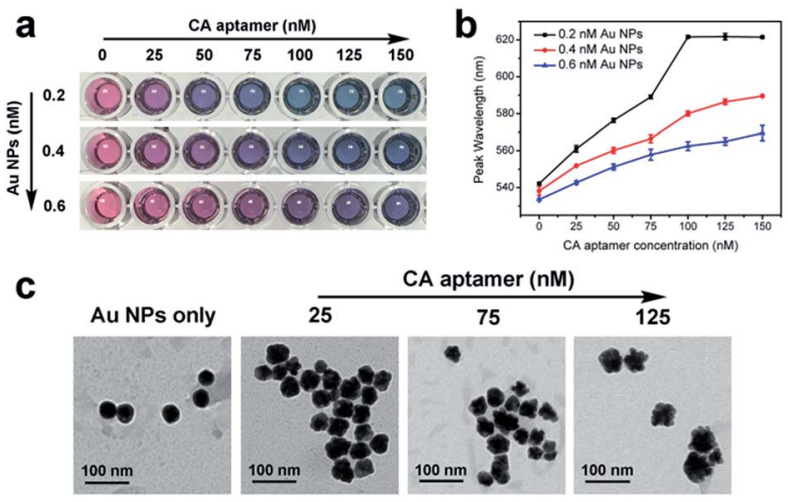

Fig. 3 (a) Photographs showing the color change after the growth reaction with increasing concentrations of $C A$ aptamers $(0,25,50,75$, 100, 125 and $150 \mathrm{nM})$ and Au NPs (0.2, 0.4 and $0.6 \mathrm{nM})$ added. (b) Corresponding LSPR shifts in the grown Au NP solution with an increasing concentration of aptamers and Au NPs. Error bars indicate the SD of three independent experiments. (c) TEM images of grown Au NPs with an increasing concentration (25, 75 and $125 \mathrm{nM}$ ) of CA aptamers adsorption, the Au NPs concentration was $0.4 \mathrm{nM}$. Scale bars $=100 \mathrm{~nm}$.

nanoparticle aggregation leading to the color change, Chávez et al. reported a method to detect four kinds of molecules, including cholic acid. ${ }^{29}$ He et al. reported a liquid crystal based sensors to detect the cholic acid molecule. ${ }^{30} \mathrm{Wu}$ et al. designed a molecularly imprinted photonic hydrogels for CA detection. ${ }^{31}$ Herein, we designed a simple and fast aptamer-Au NPs biosensor for the in vitro naked eye detection of cholic acid. The above four methods have been compared in Table 1. The mechanism for the colorimetric detection of CA was shown in Fig. 4a. In the presence of CA, specific aptamer-target interaction led to desorption of aptamers from the Au NPs surface. On the NPs, the higher concentration of CA, the lower amounts of adsorbed aptamers. Then, addition of $\mathrm{NH}_{2} \mathrm{OH} \cdot \mathrm{HCl}$ and $\mathrm{HAuCl}_{4}$ will trigger the homogeneous growth of $\mathrm{Au}$ NPs, and low aptamer coverage induced formation of the spherical-shaped Au NPs, with resultant red-color solutions. And high aptamer coverage caused grown Au NPs to exhibit a flower-like morphology, with resultant blue-color solutions. In summary, the morphology of grown Au NPs changes from flower-like to spherical NPs with the increasing concentrations of CA, along with a gradient color change of solutions from blue to purple, and finally to red. Such morphological and color changes can be characterized by a LSPR shift of grown Au NPs.

The ratio $1: 250$ of Apt-Au NP probes were adapted to detect $\mathrm{CA}$ in vitro. The $\mathrm{NH}_{2} \mathrm{OH} \cdot \mathrm{HCl}$ and two aliquots of $\mathrm{HAuCl}_{4}$ were added to trigger the growth reaction of Apt-Au NP probes after CA samples incubation for $20 \mathrm{~min}$. The increasing CA concentration caused grown Au NPs to change from blue to red gradually (Fig. 4b), accompanied by a corresponding blue shift of the UV-vis spectra (Fig. 4c). Then a dose-response curve could be achieved with the LSPR shift (Fig. 4d). The linear relationship between the CA concentrations and the LSPR shift was shown in the inner Fig. $4 \mathrm{~d}$. The linear range was from 1 to $15 \mu \mathrm{M}\left(r^{2}=\right.$ $0.9884)$. 
Table 1 Comparison of different detection methods of CA

\begin{tabular}{lll}
\hline Methods & Advantages & Disadvantages \\
\hline Chávez & Convenient; fast & Low sensitivity; LOD was larger than 10 $\mu$ M; false positive \\
He's & Facile; fast & Interference factors such as pH and ionic strength \\
Wu's & High sensitivity; good specificity; can be recycled & The result cannot be observed visibly \\
Ours & Facile; low-cost; fast; the result can be observed visibly & Interference factor such as similar molecules to CA
\end{tabular}

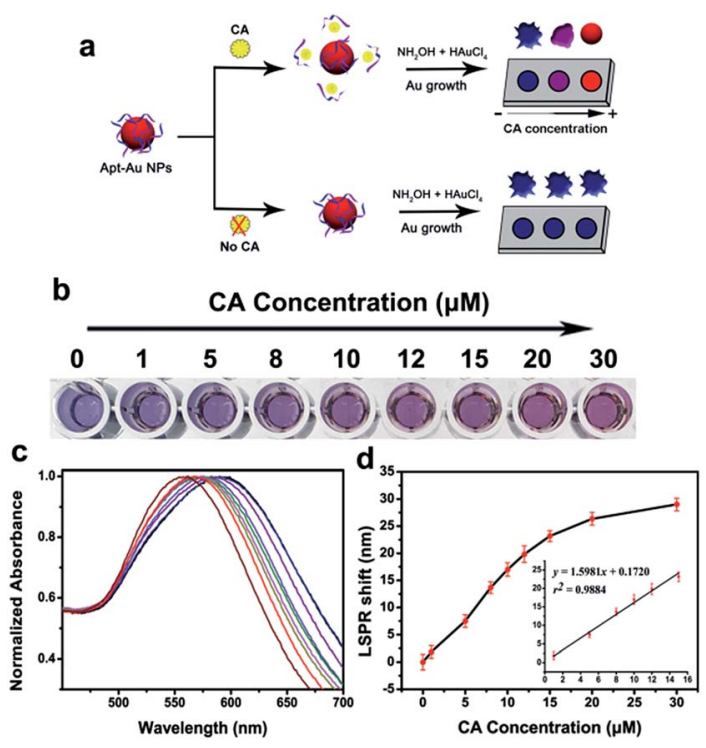

Fig. 4 (a) Schematic illustration of the mechanism for the colorimetric detection of CA. (b) Photographs of the colorimetric detection results of CA samples $(0,1,5,8,10,12,15,20$, and $30 \mu \mathrm{M})$ with the Apt-Au NP probe ratios $(1: 250)$ of Au NPs and aptamers. (c) UV-vis spectra of grown Au NPs. (d) LSPR shifts of grown Au NPs were plotted in the concentrations ranging of $0-30 \mu \mathrm{mol} \mathrm{L}{ }^{-1} \mathrm{CA}$ with the inner linear range from $1-15 \mu \mathrm{mol} \mathrm{L}^{-1}$.

To further study the detection limit of CA, we set another group with different CA concentration $(0,0.1,1$ and $10 \mu \mathrm{M})$. The increasing CA concentration caused grown Au NPs (Fig. 5a). Additionally, increasing amounts of CA concentration gave rise to a more regular shape of grown Au NPs from TEM images (Fig. 5b). The UV-vis spectra was shown in Fig. 5c. The LOD was determined to $1 \mu \mathrm{M}$ by reference to 3 times the blank SD (Fig. 5d). The detection limit can be improved by decreasing the particle size of gold nanoparticles and adjusting the ratio of $\mathrm{Au}$ nanoparticles and aptamers.

To further confirm that the $1: 250$ ratios of $\mathrm{Au}$ NPs and aptamers was the best choice for CA detection, the ratios $1: 312.5$ and $1: 375$ were set as controlling experiments. In the case of $1: 250$, increasing CA concentration caused grown $\mathrm{Au}$ NPs to change from blue to red gradually (Fig. 6a), accompanied by a corresponding blue shift of the UV-vis spectra (Fig. 6b). Then a dose-response curve could be achieved with the LSPR shift (Fig. 6e), from which the LOD was determined to $1 \mu \mathrm{M}$ by reference to 3 times the blank SD. However, it is worth noting that larger amounts of aptamers led to lower detection sensitivity of CA. From the colorimetric results, a Apt-Au NP with ratio of $1: 312.5$ and $1: 375$ caused the grown Au NPs solution bluer compared with the blank after adding a small amount of CA, and a red shift of the UV-vis spectra was witnessed (Fig. 6c and $\mathrm{d}$ ), which produced a higher LOD ( $5 \mu \mathrm{M}$ for $1: 312.5$ and 10 $\mu \mathrm{M}$ for $1: 375$, Fig. $6 \mathrm{f}$ and $\mathrm{g}$ ). Therefore, $1: 250$ was the optimal ratio of Apt-Au NP for CA detection.

Initially, the morphology of grown Apt-Au NPs was highly irregular. As the concentration of CA increased, grown Apt-Au NPs became more spherical, with an increasingly smooth surface. And grown Apt-Au NPs in the solution containing $10 \mu \mathrm{M}$ CA displayed a spherical morphology and appeared more similar to grown $\mathrm{Au}$ NPs without aptamer adsorption. Hence, LSPR shift of the solution was due to localized low-energy plasmon at the tips of the flower-like morphology rather than particle aggregation along with the particle diameter analysis of grown Apt-Au NP (Fig. S6†). Moreover, free aptamers did not influence the growth reaction as well from the control experiment (Fig. S7 $\dagger$ ).

To determine the desorption process of aptamers on the surface of Au NPs via aptamer-target affinity, we used a fluorophore (FAM) labeled CA aptamer for Apt-Au NP preparation. The fluorescence analysis showed that the addition of CA to the Apt-Au NP probe caused aptamer desorption from the Au NP surface (Fig. S8a $\dagger$ ). Increasing concentrations of CA caused fluorescence recovery of FAM, before that, the fluorescence of

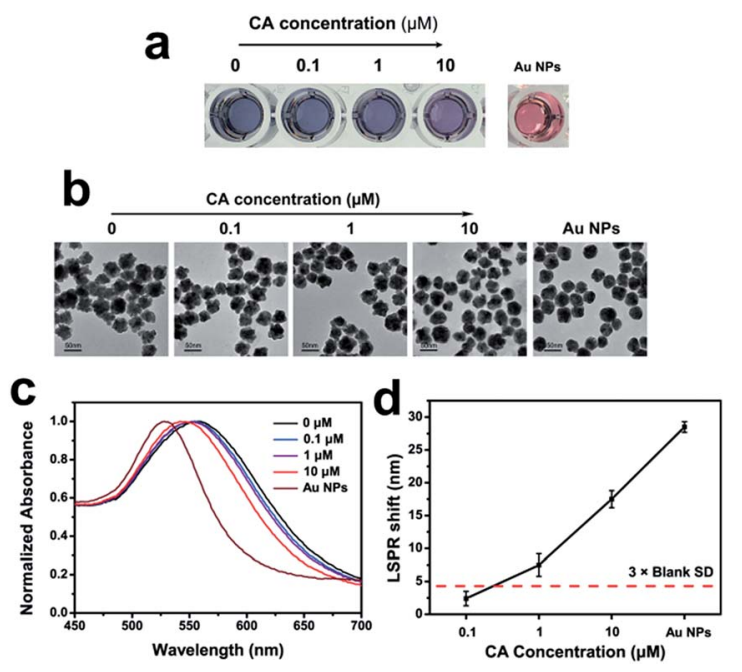

Fig. 5 (a) Photographs of the colorimetric detection results of $C A$ samples $(0,0.1,1$ and $10 \mu \mathrm{M})$. (b) TEM images of grown Au NPs coincubated with an increasing concentration $(0,0.1,1$ and $10 \mu \mathrm{M})$ of $C A$. Scale bars $=50 \mathrm{~nm}$. (c) UV-vis spectra of grown Au NPs. (d) LSPR shifts of grown Au NPs. Error bars indicate the SD of three independent experiments. 


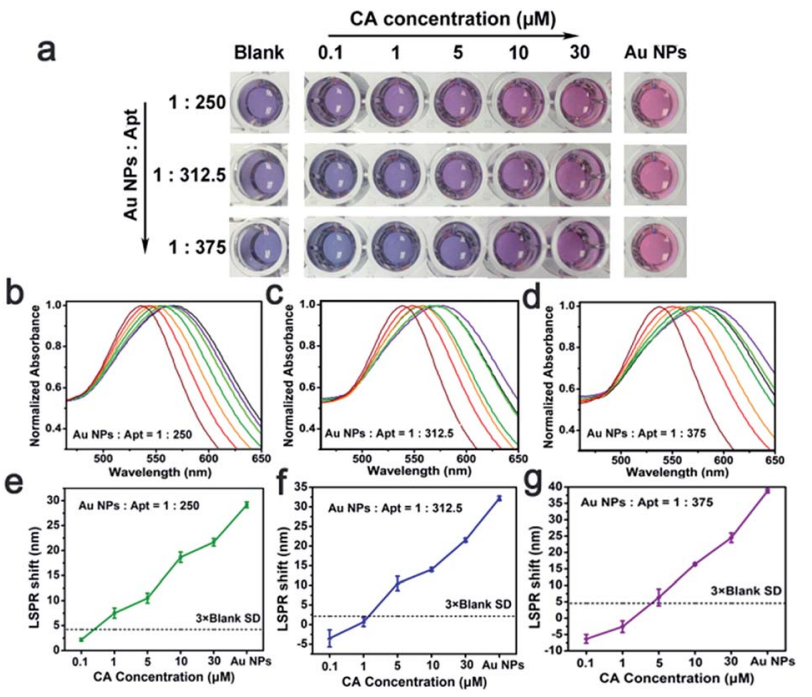

Fig. 6 (a) Photographs of the colorimetric detection results of $C A$ samples $(0.1,1,5,10$ and $30 \mu \mathrm{M})$ with the Apt-Au NP probe at different ratios (1:250,1:312.5 and $1: 375)$ of Au NPs and aptamers. (b-d) UVvis spectra of grown Au NPs corresponding to different ratios. $(e-g)$ LSPR shifts of grown Au NPs corresponding to different ratios. Error bars indicate the SD of three independent experiments.

FAM-labeled CA aptamer absorbed on Au NPs was quenched by FRET effect. ${ }^{32,33}$ To eliminate the influence of $\mathrm{pH}$ on the fluorescence intensity, FAM-Apt was treated with increasing concentrations of CA solution, and the change of fluorescence intensity was determined by a multi-mode microplate reader. As shown in Fig. S8b, $\uparrow \mathrm{CA}$ in water solution was acidulous and the $\mathrm{pH}$ tailed off to $87 \%$ compared with double distilled water as 10 $\mu \mathrm{M}$. Correspondingly, fluorescence intensity of FAM-Apt declined $14 \%$ (Fig. S8c $\dagger$ ) owing to the cationic, neutral and anionic forms of fluorescein in aqueous solution, ${ }^{34,35}$ which was weaker than the desorption effect. Furthermore, in order to investigate the possibility of nonspecific interactions between CA and Au NPs, the Au NP growth reaction was conducted after incubating bare Au NPs with different concentrations of CA. It showed negligible LSPR shifts (Fig. S9†) of re-growth particles even at the highest CA concentration $(10 \mu \mathrm{M})$, indicating that CA did not affect the re-growth of Apt-Au NP. For control, the solution became dark in blue after addition of complementary DNA sequence of CA aptamer (Fig. S10 + ), indicating that the complementary sequence hybridized to the aptamer and increased the amount of total DNA absorbed on the Au NPs surface, which induced more irregular growth of Au NPs. These results demonstrated that only addition of targets could cause the desorption of aptamers from the surface of Au NPs via aptamer-target binding.

\section{Selectivity of detection}

Four fairly similar molecules from the family of bile acid were detected as well to testify the specificity of the sensor (DCA, CDCA, GCA and LCA, structures shown in Fig. S11†). A similar phenomenon (Fig. S12 $\dagger$ ) of color change and LSPR shift as different concentrations of the family of bile acid were added to the Apt-Au NP probes. Increasing the concentration of DCA and CDCA caused grown Au NPs to change from blue to red color, accompanied by a corresponding blue shift $(\sim 25 \mathrm{~nm})$ of LSPR spectra. And GCA by contrast, was shown a lower response by the probe with $\sim 16 \mathrm{~nm}$ LSPR shift at high concentration (10 $\mu \mathrm{M})$. Nevertheless, even much lower sensitivity was observed at higher LCA concentrations $(10 \mu \mathrm{M})$ both from color and LSPR results. The selectivity can be enhanced by using aptamer with stronger affinity for cholic acid.

\section{Conclusions}

In summary, we have demonstrated a versatile gold nanoprobe for rapid colorimetric detection of CA based on the aptamer-target recognition and the aptamer controlled growth of Au NPs. In this work, we could visually detect as low as $1 \mu \mathrm{M}$ CA in an hour without any sophisticated instrument or highly qualified personnel. The LOD was lower than the physiological limits of CA and the sensitivity was higher than the existing colorimetric detection method for CA based on Au NPs, which was resulted from the mechanism that even small differences in the amount of adsorbed aptamer could produce morphologically significant variations in grown Au NPs with visible color changes and obvious LSPR shifts. Furthermore, we verified the affinity between CA and $48 \mathrm{nt}$ aptamer $\left(K_{\mathrm{d}}=12.6 \pm 0.695 \mu \mathrm{M}\right)$ for the first time by MST technology, which is reconcilable compared with the results from equilibrium-filtration method as reported. This research shows that nanoprobe can detect an array of bile acids rapidly and making it applicable for combined diagnosis. Given the advantages of colorimetric assay based on Au NPs, the assay is wellpoised as a POC tool for the naked eye detection of CA.

\section{Notes}

The authors declare no competing financial interest.

\section{Acknowledgements}

The authors are grateful to National Basic Research Program of China 973 Program Grant No 2015CB755500 and Natural Science Foundation Committee of China (NSFC 81220108012, 61335007, 81371684, 81000666, 81171395 and 81328012) for their financial support.

\section{Notes and references}

1 S. Mukhopadhyay and U. Maitra, Curr. Sci., 2004, 87, 16661683.

2 C. Huang, Y. Guo and J. Yuan, Poult. Sci., 2014, 93, 14751483.

3 K. Rani, P. Garg and C. S. Pundir, Anal. Biochem., 2004, 332, 32-37.

4 W. J. Griffiths and J. Sjovall, J. Lipid Res., 2010, 51, 23-41.

5 I. Burkard, A. von Eckardstein and K. M. Rentsch, J. Chromatogr. B: Anal. Technol. Biomed. Life Sci., 2005, 826, 147-159. 
6 Y. Wang, J. Zhang, X. X. Zhu and A. Yu, Polymer, 2007, 48, 5565-5571.

7 S. He, W. Liang, C. Tanner, K.-L. Cheng, J. Fang and S.-T. Wu, Anal. Methods, 2013, 5, 4126-4130.

8 K. M. Mayer and J. H. Hafner, Chem. Rev., 2011, 111, 38283857.

9 Y. Zhang, Y. Tang, Y. H. Hsieh, C. Y. Hsu, J. Xi, K. J. Lin and X. Jiang, Lab Chip, 2012, 12, 3012-3015.

10 E. Hutter and J. H. Fendler, Adv. Mater., 2004, 16, 1685-1706.

11 W. Zhou, X. Gao, D. Liu and X. Chen, Chem. Rev., 2015, 115, 10575-10636.

12 S. J. Chen, Y. F. Huang, C. C. Huang, K. H. Lee, Z. H. Lin and H. T. Chang, Biosens. Bioelectron., 2008, 23, 1749-1753.

13 F. Xia, X. Zuo, R. Yang, Y. Xiao, D. Kang, A. Vallée-Bélisle, X. Gong, J. D. Yuen, B. B. Hsu and A. Heeger, Proc. Natl. Acad. Sci. U. S. A., 2010, 107, 10837-10841.

14 D. Liu, J. Yang, H. F. Wang, Z. Wang, X. Huang, Z. Wang, G. Niu, A. R. Hight Walker and X. Chen, Anal. Chem., 2014, 86, 5800-5806.

15 J. N. Anker, W. P. Hall, O. Lyandres, N. C. Shah, J. Zhao and R. P. Van Duyne, Nat. Mater., 2008, 7, 442-453.

16 M. J. Taussig, O. Stoevesandt, C. A. Borrebaeck, A. R. Bradbury, D. Cahill, C. Cambillau, A. de Daruvar, S. Dübel, J. Eichler and R. Frank, Nat. Methods, 2007, 4, 13-17.

17 E. J. Cho, J. W. Lee and A. D. Ellington, Annu. Rev. Anal. Chem., 2009, 2, 241-264.

18 J. Wang, Q. Gong, N. Maheshwari, M. Eisenstein, M. L. Arcila, K. S. Kosik and H. T. Soh, Angew. Chem., 2014, 53, 4796-4801.

19 J. Wang, J. Lu, S. Su, J. Gao, Q. Huang, L. Wang, W. Huang and X. Zuo, Biosens. Bioelectron., 2015, 65, 171-175.
20 H. Jiao, J. Chen, W. Li, F. Wang, H. Zhou, Y. Li and C. Yu, ACS Appl. Mater. Interfaces, 2014, 6, 1979-1985.

21 F. Wang, S. Liu, M. Lin, X. Chen, S. Lin, X. Du, H. Li, H. Ye, B. Qiu, Z. Lin, L. Guo and G. Chen, Biosens. Bioelectron., $2015,68,475-480$.

22 Z. Wang, J. Zhang, J. M. Ekman, P. J. Kenis and Y. Lu, Nano Lett., 2010, 10, 1886-1891.

23 J. H. Soh, Y. Lin, S. Rana, J. Y. Ying and M. M. Stevens, Anal. Chem., 2015, 87, 7644-7652.

24 J. J. Storhoff, R. Elghanian, C. A. Mirkin and R. L. Letsinger, Langmuir, 2002, 18, 6666-6670.

25 M. Östblom, B. Liedberg, L. M. Demers and C. A. Mirkin, J. Phys. Chem. B, 2005, 109, 15150-15160.

26 Z. D. Liu, H. Y. Zhu, H. X. Zhao and C. Z. Huang, Talanta, 2013, 106, 255-260.

27 H. Li and L. J. Rothberg, J. Am. Chem. Soc., 2004, 126, 1095810961.

28 T. Kato, T. Takemura, K. Yano, K. Ikebukuro and I. Karube, Biochim. Biophys. Acta, Gen. Subj., 2000, 1493, 12-18.

29 J. L. Chávez, J. K. Leny and S. Witt, Analyst, 2014, 139, 62146222.

30 S. He, W. Liang and C. Tanner, Anal. Methods, 2013, 5, 41264130.

31 Z. Wu, X. Hu and C. Tao, J. Mater. Chem., 2008, 18, 54525458.

32 L. Stryer and R. P. Haugland, Proc. Natl. Acad. Sci. U. S. A., 1967, 58, 719-726.

33 K. Saha, S. S. Agasti, C. Kim, X. Li and V. M. Rotello, Chem. Rev., 2012, 112, 2739-2779.

34 W. Shi, S. He, M. Wei, D. G. Evans and X. Duan, Adv. Funct. Mater., 2010, 20, 3856-3863.

35 R. Sjöback, J. Nygren and M. Kubista, Spectrochim. Acta, Part A, 1995, 51, L7-L21. 